\title{
RECENSIÓN
}

\section{CERTIFICA. AUTOFORMACIÓN MÉDICA PARA LA CERTIFICACIÓN DE DEFUNCIÓN}

Lluís Cirera, Enrique Vázquez, Sara Cerdeira, Luis Alberto Sangrador, Gloria Pérez y Covadonga Audicana.

Santiago de Compostela; 2003

Editan: Consejerías de Salud de las Comunidades Autónomas de Murcia, Galicia, Castilla y León, Cataluña, País Vasco, y Ministerio de Sanidad y Consumo

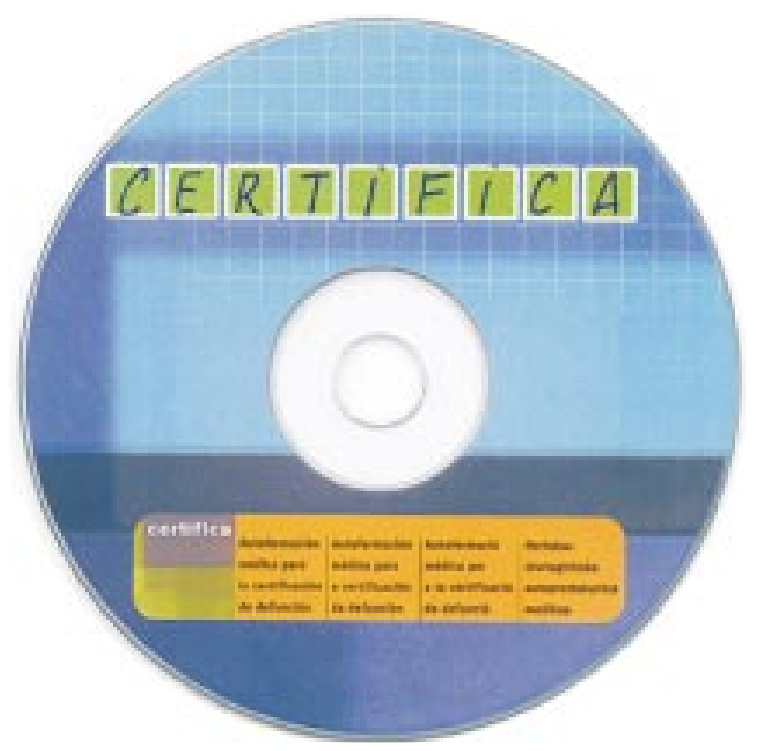

\section{¿QUÉ ES CERTIFICA?}

Hacia finales de la década de los 80 se ampliaron los convenios de colaboración para la codificación de los boletines estadísticos de defunción entre el Instituto Nacional de Estadística y los Institutos de Estadística de la Comunidades Autónomas, a la mayoría de éstas. Esta nueva situación impulsó el interés por la mejora de la calidad de los indicadores de mortalidad y por lo tanto de la certificación de la muerte. Consecuentemente, varios registros de mortalidad de Comunidades Autónomas comenzaron a impartir cursos de certificación de defunción entre los estudiantes de medicina del último año 
de carrera y a médicos, tanto de atención primaria como especializada.

En la actualidad cada vez son más los registros de mortalidad que imparten cursos sobre esta temática lo que ha dado lugar a un consenso en la percepción de la necesidad de contar con una metodología docente y materiales comunes, que homogenicen la formación de los médicos y en definitiva favorezcan el objetivo de mejora de calidad y la comparabilidad de las estadísticas de mortalidad.

En este contexto nace el proyecto CERTIFICA, que trata de satisfacer esta demanda por dos vías. Por un lado, a través de las 5 unidades didácticas proporciona al docente material sobre aspectos jurídicos y documentos, sobre la importancia y usos de las estadísticas de defunción y un banco de casos, basados en la experiencia real de los registros de mortalidad, para que se ejerciten los alumnos. También facilita al docente una propuesta de organización del curso y un formulario de evaluación.

CERTIFICA ofrece por otra parte la posibilidad de utilizarse como un programa de autoaprendizaje. La presentación de los materiales en un CD que incluye una sencilla aplicación para el manejo del banco de casos prácticos, permite al interesado en el tema repasar los aspectos teóricos y las normas, diseñar sus ejercicios, comprobar las respuestas razonadas y registrar su propia estadística de aciertos y errores.

\section{MÓDULOS DE CERTIFICA}

El programa CERTIFICA consta de una presentación, de un Decálogo, y de cinco Unidades Didácticas.

- Así, el Decálogo enumera las normas básicas que el médico certificador debe tener presentes para la correcta certificación de una defunción al rellenar los impresos oficiales.
- La Unidad Didáctica de Información Jurídico-Legal revisa los aspectos de la legislación española relacionados con la certificación de los fallecimientos que más pueden concernir al profesional médico. También aporta al lector información sobre el procedimiento judicial ante una muerte accidental o violenta. Tiene como objetivos:

- Informar al profesional médico de la normativa legal vigente en España.

- Diferenciar los distintos tipos de autopsias y sus indicaciones.

- Caracterizar el papel de los Juzgados de Instrucción en la certificación de la muerte no natural.

- La unidad Didáctica 2 de CERTIFICA tiene como objetivo presentar de forma esquemática los usos más habituales y las limitaciones de las estadísticas de mortalidad. Subraya la importancia de disponer de datos de calidad para producir una información pertinente y de utilidad y, en definitiva, aclarar por qué se necesitan y para qué se usan los datos que el clínico produce sobre mortalidad. Esta unidad docente se divide en cuatro apartados. El primero es de carácter genérico sobre utilidad y limitaciones de las estadísticas de mortalidad. El segundo apartado relaciona, con una explicación breve, los distintos métodos que habitualmente se utilizan en los análisis de mortalidad. El tercero explica de forma simple los métodos de presentación de resultados más comunes y el cuarto muestra una serie de publicaciones periódicas sobre mortalidad, tanto internacionales, como de España y de sus Comunidades Autónomas.

- Unidad de cumplimentación de los Documentos. La certificación de la defunción es un acto médico jurídico- 
administrativo que permite, por una parte, el enterramiento del fallecido, y por otra, conocer el patrón de las causas de muerte en una comunidad. Para habilitar estos dos aspectos, en la actualidad, se deben de cumplimentar una serie de documentos oficiales, que recorren unos circuitos que esta unidad describe. Siendo los objetivos de la unidad: describir los documentos oficiales utilizados para la certificación de defunción, la forma correcta de cumplimentación, enfatizando los que corresponden al médico, y explicar el circuito administrativo que recorren los documentos.

- La Unidad de Ejercicios de Certificación tiene como objetivo facilitar el aprendizaje de los conceptos revisados en las unidades Júrido-Legal y de Cumplimentación de los Documentos, mediante la realización de ejemplos prácticos. Para ello hemos desarrollado una aplicación informática que permite la elaboración, realización y corrección de ejercicios por parte del alumno. Estos ejercicios se crean a partir de los 176 casos elaborados por el grupo de trabajo del CERTIFICA. El usuario puede generar aleatoriamente su ejercicio a partir del banco de casos o seleccionar aquellos que considere adecuados a sus objetivos. Aunque este módulo de ejercicios es intuitivo y de manejo sencillo ante cualquier duda sobre su funcionamiento puede consultar la ayuda dentro del propio programa.

- El módulo de la Sesión Docente tiene como objetivos, el proporcionar herramientas docentes probadas a los encargados de la formación para impartir clases presenciales, en la mejora en la cumplimentación de los boletines estadísticos de defunción (fallecidos con un día o más de vida) y de parto (nacidos muertos y nacidos vivos que fallecen antes del día); y el de homogeneizar los métodos de enseñanza en esta materia. 